\title{
Customer Requirements for a Vitreoretinal Robot
}

\author{
Xu HAN ${ }^{1, a,{ }^{*}}$, Yang YANG ${ }^{1, b}$ \\ ${ }^{1}$ Department of Mechanical Design and Automation, Beihang University, Beijing, 100191, China \\ ahaxconan@gmail.com, byang_mech@buaa.edu.cn \\ ${ }^{*}$ Corresponding author
}

\begin{abstract}
Keywords: Customer requirement, Vitreoretinal robot, House of Quality (HOQ), Degree of importance.
\end{abstract}

\begin{abstract}
Several vitreoretinal surgical robots have been developed for specific tasks. However, there is no complete methodology for analysing customer requirements in the initial design. This paper focused on customer requirements for a vitreoretinal surgical robot and analysed the degree of importance for each requirement in the House of Quality model. Validations were provided to assess its feasibility. Based on the results of validation, there are some disagreements about some requirements, because of different concerns in these two groups. As vitreoretinal robot is a new product with limited information, the results need to be improved in the future.
\end{abstract}

\section{Introduction}

According to statistics of World Health Organisation published in the years of 2002 and 2010, the percentages of people with visual impairment increased from $2.6 \%$ to $4.2 \%$. Vitreoretinal surgery is a typical method to solve several ophthalmologic problems, such as retinal detachment, macular pucker, macular hole and diabetic retinopathy [1]. Taking account of growing demands for vitreoretinal surgery, robots for assisting retinal surgery have been proposed.

Vitreoretinal surgery is an ophthalmological task, working on small and delicate ocular tissues. It is one of the most challenging surgical tasks because of its weak operability of sophisticated surgical facilities, surgeons' physiological hand tremor, inability to regenerate if injured, limited visualisation and constraint accessibility to some areas [2,3]. Differently from some ordinary surgeries, vitreoretinal surgery is a microsurgery that requires a considerable high demand of precision. It is stated that precision is approximately $10 \mu \mathrm{m}$ [4], but physiological hand tremor amplitude under surgical conditions is 182 $\mu \mathrm{m}$ [5]. Only a few surgeons can successfully manipulate these operations [4]. In order to achieve better clinical effects with more surgeons, surgical assistant robots are required for vitreoretinal surgery.

Surgical assistant robot is not to take the place of surgeon in the surgery, but to optimize the capability of multi-tools or to extend surgeons' ability to operate [7]. Based on roles during robotic surgery, surgical assistant robots can be divided into two aspects: auxiliary surgical supports and surgeon extenders [6]. The former, working aside with surgeons, provides functions including holding and retracting endoscope or microscope. These robots have been succeed in experiments and come into service. For instance, assistant robot for laparoscopic camera manipulation is commonly used currently [7]. The latter, directly operated by surgeons, is to lift capability of surgical instrument manipulations [6]. A large number of literatures have researched these surgeon extenders, such as Da Vinci system [9], which has been used in laparoscopic surgery [2], and robots from John Hopkins University (JHU) [1,6]. Jensen et al. designed an ophthalmic surgery manipulator for retinal vascular microsurgery. However, it was not suitable for micro-surgical applications due to the lack of force sensing and hand tremor [9].

Several institutes and companies have developed kinds of vitreoretinal robots, according to specific tasks or experiments [2,5]. However, there is no complete methodology for analysing customer requirements in the initial design of these robots. Vitreoretinal robot as a product, it is vital to meet more customer needs. Ophthalmologists, ophthalmic patients and robotic designers are customers for vitreoretinal robots. Because of different concerns between these customers, it is difficult to guarantee the consistency of design targets and customer requirements. Combining with the current situation that no general method for collecting customer requirements of a vitreoretinal surgical robot at the initial design stage, 
information omission might be led during translations from customer needs to technical measures [10]. With the market expansion of vitreoretinal robots, customer requirements are increasingly important on the robot development. A high level of customer satisfaction can get higher loyalty from customers and greater market scale. Therefore, customer requirement is not only the basis of new product design, but also a positive factor for product development in the future.

Quality Function Deployment (QFD) is a decision-making technique, with 'voice of the customer' as its philosophy and driver. It converts customer needs into design factors of products and processes step by step. It is applied from upstream to downstream of a product, avoiding quality problems in the production stage. Owing to several benefits, such as reduction of development cycles, cost reduction and quality improvement, QFD provides not only better customer satisfaction but greater productivity for a general vitreoretinal surgical robot. House of Quality (HOQ) as the first step of QFD is the basis in this technique. It assembles and analyses multidisciplinary customer requirements, satisfying the maximum number of needs and also identifies the relationships between requirements or measures. HOQ model have been used in some designs of medical device [11]. However, customer requirements of vitreoretinal robot have not been discussed in this method.

In this paper, it focuses on customer requirements for a vitreoretinal surgical robot and gives a methodology for collecting and analysing them. HOQ model is applied to implement and validations for degree of importance are provided from robot designers and ophthalmologists.

\section{Method and Materials}

House of Quality has a matrix including a vertical column of WHATs and a horizontal row of HOWs [10]. WHATs are defined as customer requirements and HOWs are technical requirements, which are the ways to achieve WHATs. In an original HOQ, there are basically seven parts in the model: Customer requirements (CRs), Technical requirements (TRs), Target, Relationships, Degree of Importance, Absolute \& Relative Demanded Weight and Technical Importance [12]. According to the needs of this research, five aspects of modification are taken into consideration.

1. Classification of customer requirements

Large amount of unordered data increase difficulties of verifying and searching information. The categories can provide convenience for validation and development of vitreoretinal surgical robots in the future. For example, 'easy to remove' and 'multi-tools' refer to 'tools' application.

2. Internal relationships between CRs

It provides the correlation of customer requirements, which described as positive relationship, weak positive relationship, weak negative relationship and negative relationship.

\begin{tabular}{|c|l|}
\hline Definition of Customer Groups & $\begin{array}{l}\text { - Ophthalmologic surgeons } \\
\text { - Vitreoretinal robot designers }\end{array}$ \\
\hline $\begin{array}{c}\text { Customer Requirements (CRs) } \\
\text { and its Degree of Importance }\end{array}$ & $\begin{array}{l}\text { - Collecting customer requirements through questionnaires } \\
\text { - Analysing internal relationships } \\
\text { - Marking the degree of importance based on literature review }\end{array}$ \\
\hline Technical Requirements (TRs) & $\begin{array}{l}\text { - Collecting technical requirements from literature } \\
\text { - Analysing internal relationships }\end{array}$ \\
\hline Relationships between & $\begin{array}{l}\text { - Searching correspondence between CRs and TRs } \\
\text { CRs and TRs } \\
(\bullet), \text { Strong relationship (O) and Weak relationship }(\circ)\end{array}$ \\
\hline Weight Calculation & $\begin{array}{l}\text { - Calculating relative weight of each customer requirement or } \\
\text { technical measure based on the value in their relationships }\end{array}$ \\
\hline
\end{tabular}

Fig. 1 Five steps of model implementation

\section{Delete target block}

Considering vitreoretinal surgical robots have not been used in the surgical operations, it is unpractical to get real operating data of previous robots. 
4. New marking method for 'degree of importance'

Considering that some CRs are partly conflicting, it is impossible to fully satisfy all requirements. Marking of CRs is a significant stage in the QFD technique. Higher degree of importance provides higher concentration of customers, related to higher customer satisfaction. Therefore, it is important for designers to sort importance of CRs in order to obtain higher customer satisfaction, with achieving prioritized requirements. In this method, degree of each customer requirement shows its awareness or impact during product design. Precisely, frequency of occurrence for each CR in literature is calculated, then translated into percentage of all customer requirements. And finally, it is shown as a number from 1 to 10 , representing its interval value.

5. Customer competitive assessment

In order to ensure availability of this HOQ model, validation for degree of importance is provided by different groups of customers. The final proposed model is in Fig. 1. Its five steps of implementation are shown in Fig. 2.

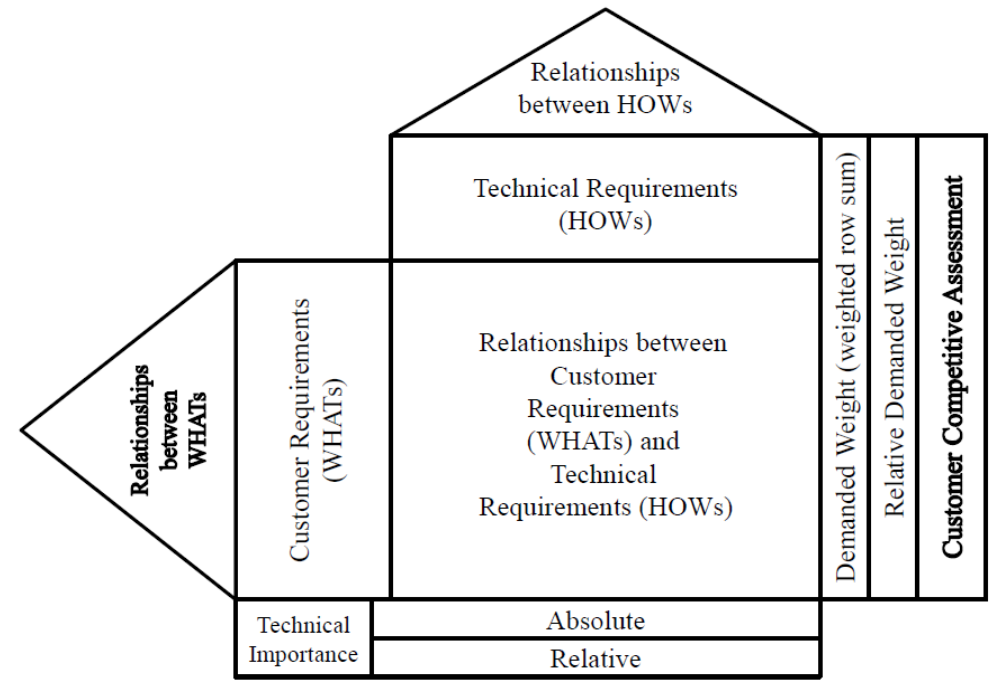

Fig. 2 Proposed House of Quality Model

Validation evaluates the application value of HOQ model. In addition, validation is a necessity to identify the different views between literature and experts. HOQ validation is commonly in two ways: observation after product being into market and assessment from experts. The former is suitable for some small or simple product, therefore, the latter approach is chosen in this research. There are four parts of validation for HOQ model: Customer Requirements, Technical Requirements, Degree of Importance and Relationships.

Three customer groups of patients, ophthalmologists and designers are considered initially in the HOQ model building. However, retinal patients, as potential customers have no knowledge of specific operations of vitreoretinal robot, it is pointless for patients to do validations. Considering that some designers of vitreoretinal robots have been researched these robots for years, they are familiar with vitreoretinal robots. Ophthalmologists are undoubtedly those who can best understand customer requirements and also the end users of this product. In brief, two validation groups are selected as the experts of this research. Taking account of distinct emphases in these two customer groups, validations are divided into two separated groups. Therefore, ten designers of vitreoretinal surgical robots and a group of retinal surgeons are chosen to be the validators.

\section{Results}

As it shows in Table 1, there are twenty-three customer requirements and twenty-three technical requirements summarized in the HOQ model. Precision and safety are the most important customer requirements, receiving the highest rating of 10 on the scale from 1 to 10 . On the other hand, needs of price and shorten setup $\&$ operating time are paid less attention, rating the lowest importance from the 
literature. In addition, constraint motion should also be given priority from the result of demanded weight calculation, as its high weight.

In reference to technical requirements, virtual fixture and coarse-fine adjustment can be applied to achieve multi-objective in terms of the relationships between customer and technical requirements. Also, virtual fixture represents larger proportions, showing its significantly importance among these requirements. After analysing, its characteristic is that it plays vital roles on various high priority customer requirements. It is more favourable to apply multi-functional technical measures. On the contrary, lengthen manipulator has less importance, illustrating that this solution may provide less improvement value on vitreoretinal assistant robot.

Data from questionnaires and its analysis are shown in Table 2. From the views of vitreoretinal robot designers, precision and safety are as important as they are in the literature. Nevertheless, importance rating for mechanism stability, good visualisation, collision avoidance and compatibility with surgical environment is comparably increased. The score of positioning control has a $25 \%$ reduction compared to that in the literature, while price and control sharing still keep a low priority. Some designers suggested adding some technical issues, such as damping platform and end-effectors to improve dexterity and stability of vitreoretinal robot.

Table 1. House of Quality

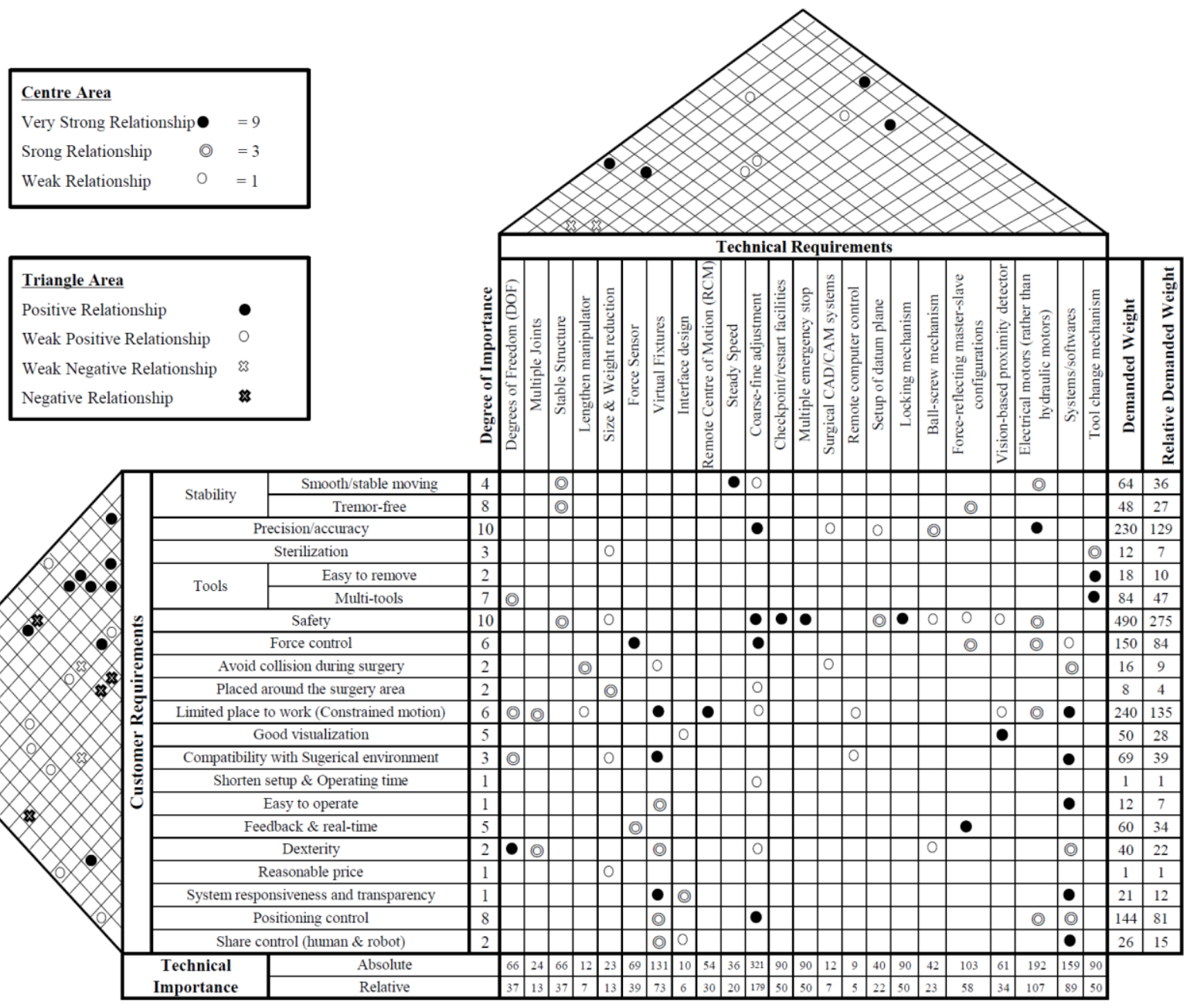

For retinal surgeons, precision, sterilization, safety, collision avoidance and placing around surgical area are marked as the highest degree of importance. Constraint motion, feedback, dexterity and positioning control are ranked only next to the previous ones. As a new product, there is less limitation to its price. With the development of computer control technology, remote control robots have been widely used in the industrial areas. Though this technology has not been succeed in the vitreoretinal surgical field, it is an inevitable development trend in the future. 
Table 2. Validations

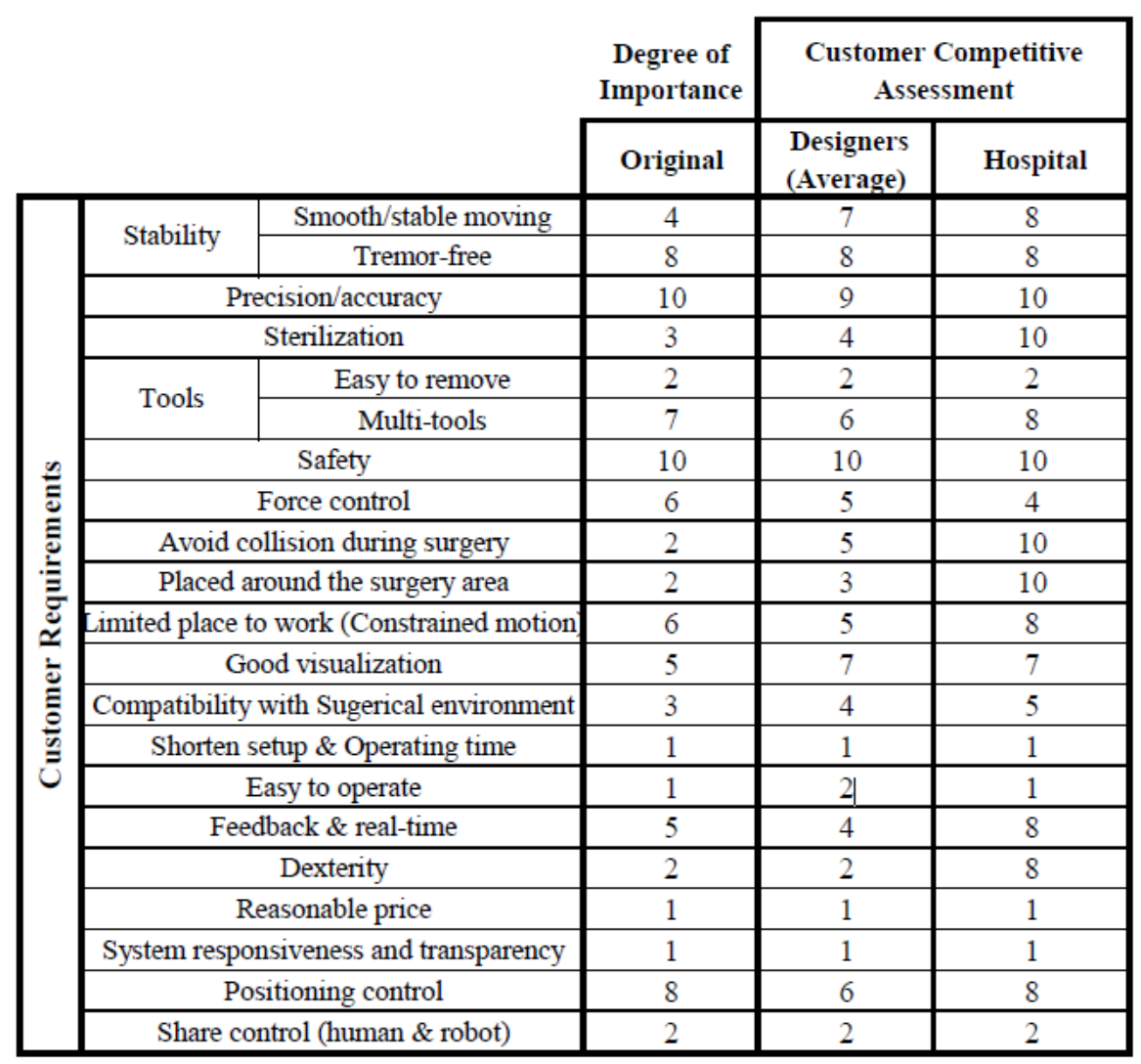

\section{Discussions}

Most markings for degree of importance in the literature are similar with results from designers in the validation. However, there is a strong disagreement about importance degree of some customer requirements between literature and validation. The reason for this situation can be different concerns from diverse customer groups. Surgeons pay fully attention to the operational performance in the surgical procedures than specific details. However, designers need to concentrate on not only detail implementation, but usability of these robots. In this situation, the designers give a uniform evaluation on the degree of importance for customer requirements, whereas data from retinal surgeons express a nearly polarization of importance.

As vitreoretinal robot is a new product on the market, information limitation makes it difficult to collect customer requirements. Furthermore, customer demands collected from literature review, which represents more opinions of designers, lead a gap between the markings of requirements from designers and surgeons.

\section{Conclusion}

The objectives of this research are focusing on customer requirements, using House of Quality model. Considering the needs of this research, HOQ has been modified with new marking system of occurrence frequency for each requirement. After analysis of results, there are some differences between two groups of validations, because of different concerns about these robots. In this paper, it provides a new approach to mark the degree of importance and a comprehensive collection of customer requirements for a vitreoretinal surgical robot. It is beneficial to improve customer satisfaction. However, vitreoretinal robot has not been familiar to customers as a new product. There are some limitations on the collection and evaluation of customer requirements.

In the future, face-to-face meeting between designers and surgeons are increasingly significant to reduce the misunderstanding between these groups. With the development of vitreoretinal surgical robot, they will be introduced to market and clinical tasks. With these experiments and applications, Failure 
Mode and Effects Analysis (FMEA), which focuses on solving potential problems, can be combined with QFD to improve the design of robot. Other functions of HOQ model can also be added into the current model, such as the competitive benchmark with other similar product.

\section{Acknowledgements}

The program is supported by National Natural Science Foundation of China (Grant No. 51175013), Ministry of Health Research Foundation of Zhejiang (WKJ2010-2-018) and Major Issue Funded Project of Eye hospital of Wenzhou Medical College (YNZD201003).

\section{References}

[1] Fleming, I., Balicki, M., Koo, J., Iordachita, I., Mitchell, B., Handa, J., Hager, G. and Taylor, R., (2008), Cooperative robot assistant for retinal microsurgery.

[2] Üneri, A., Balicki, M. A., Handa, J., Gehlbach, P., Taylor, R. H. and Iordachita, I. (2010), "New steady-hand eye robot with micro-force sensing for vitreoretinal surgery", $20103 \mathrm{rd}$ IEEE RAS and EMBS International Conference on Biomedical Robotics and Biomechatronics, BioRob 2010, pp. 814.

[3] Ida, Y., Sugita, N., Ueta, T., Tamaki, Y., Tanimoto, K. and Mitsuishi, M. (2012), "Microsurgical robotic system for vitreoretinal surgery", International Journal of Computer Assisted Radiology and Surgery, vol. 7, no. 1, pp. 27-34.

[4]Nakano, T., Sugita, N., Ueta, T., Tamaki, Y. and Mitsuishi, M. (2009), "A parallel robot to assist vitreoretinal surgery", International Journal of Computer Assisted Radiology and Surgery, vol. 4, no. 6, pp. 517-526.

[5] Singh, S. P. N. and Riviere, C. N. (2002), "Physiological tremor amplitude during retinal microsurgery", Bioengineering, Proceedings of the Northeast Conference, pp. 171.

[6] Taylor, R. H. and Stoianovici, D. (2003), "Medical robotic systems in computer-integrated surgery", Problems in General Surgery, vol. 20, no. 2, pp. 1-9.

[7] Faraz, A. and Payandeh, S. (1997), "Robotic case study: Optimal design for laparoscopic positioning stands", Proceedings - IEEE International Conference on Robotics and Automation, Vol. 2, pp. 1553.

[8] Ghodoussi, M., Butner, S. E. and Wang, Y. (2002), "Robotic surgery - The transatlantic case", Proceedings - IEEE International Conference on Robotics and Automation, Vol. 2, pp. 1882.

[9] Jensen, P. S. (1997), "Toward robot-assisted vascular microsurgery in the retina", Graefe's Archive for Clinical and Experimental Ophthalmology, vol. 235, no. 11, pp. 696-701.

[10] Dale, B. G. (1999), Managing quality / Barrie G. Dale, Oxford: Blackwell, 1999; 3rd ed.

[11] Temponi, C., Yen, J. and Tiao, W. A. (1999), "House of quality: A fuzzy logic-based requirements analysis", European Journal of Operational Research, vol. 117, no. 2, pp. 340-354.

[12] Wasserman, G. S. (1993), "On how to prioritize design requirements during the QFD planning process", IIE transactions, vol. 25, no. 3, pp. 59-65. 